\title{
EFEITO DE DIFERENTES PONTAS E PRESSÕES DE PULVERIZAÇÃO NA APLICAÇÃO DE CARBONATO DE CÁLCIO EM MUDAS DE CAFÉ CONILON
}

\author{
EFFECT OF DIFFERENT SPRAY TIPS AND PRESSURES ON THE APPLICATION OF CALCIUM \\ CARBONATE IN CONILON COFFEE SEEDLINGS
}

\author{
$\underline{\text { Débora Moro Soela }}^{{ }^{*}}, \underline{E d n e y ~ L e a n d r o ~ d a ~ V i t o ́ r i a ~}^{2}$, Antelmo Ralph Falqueto $^{3}, \underline{\text { Ismael }}^{6}$ \\ Lourenco de Jesus Freitas $^{4}$, Priscila Conceicão Souza Braga $^{5}, \&$ Cátia Aparecida Simon $^{6}$ \\ 123456 Centro Universitário Norte do Espírito Santo (CEUNES), Universidade Federal do Espírito Santo (UFES) \\ 1* deboramoro.s@ hotmail.com ${ }^{2}$ vitoria.edney@gmail.com ${ }^{3}$ antelmofalqueto@gmail.com \\ 4 ismaelljf@yahoo.com.br ${ }^{5}$ priscila.csbraga@gmail.com ${ }^{6}$ catiasimonsimon@gmail.com
}

\section{ARTIGO INFO.}

Recebido em: 21.07.2021

Aprovado em: 10.11.2021

Disponibilizado em: 17.11.2021

Palavras-Chave:

Cobertura foliar; gotas; espectro de gotas.

\section{KEYWORDS:}

Leaf coverage; drops; drops spectrum.

*Autor Correspondente: Soela, D. M.

\section{RESUMO}

A aplicação eficiente de produtos agrícolas depende de uma série de fatores, dentre eles a escolha correta da ponta de pulverização e pressão de trabalho. Objetivou-se com esse estudo, determinar qual o conjunto de pontas de pulverização e pressão de trabalho promovem a melhor cobertura em mudas de café conilon. $\mathrm{O}$ experimento foi conduzido em delineamento de blocos casualizados em esquema fatorial $3 \times 4$, três pontas de pulverização (BD 01, MGA $\left.60^{\circ} 1,0, \operatorname{MCP} 1,5\right)$, quatro pressões de pulverização (206, 275, 344 e $413 \mathrm{kPa}$ ), quatro repetições e quatro plantas em cada parcela. A taxa de aplicação foi a mesma em todos os tratamentos, $100 \mathrm{~L} \mathrm{ha}^{-1}$ e dose de $2 \mathrm{~L} \mathrm{ha}^{-1}$ do produto utilizado. A primeira aplicação do produto foi realizada dentro do viveiro, e as posteriores, em campo após o plantio das mudas, com intervalo de sete dias entre elas. Foram realizadas análises de espectro de gotas, onde foram avaliados os parâmetros de diâmetro da mediana volumétrica (DMV), densidade de gotas (DEN) e cobertura (COB). A variável cobertura foi influenciada pela pressão em todas as pontas, conforme aumenta a pressão, melhor a porcentagem de cobertura. A ponta cone vazio MGA $60^{\mathrm{a}}$ na pressão de $413 \mathrm{kPa}$ promoveu a melhor porcentagem de cobertura do produto avaliado, portanto, melhor opção para aplicação.

\section{ABSTRACT}

The efficient application of agricultural products depends on a number of factors, including the correct choice of spray tip and working pressure. The objective of this study was to determine which set of spray tips and working pressure provide the best coverage in conilon coffee seedlings. The experiment was conducted in a randomized block design in a factorial scheme $3 \times 4$ three spray tips (BD 01, MGA $60^{\circ}$ 1.0, MCP 1.5), four spray pressures (206, 275, 344 and $413 \mathrm{kPa}$ ), four replications and four plants in each plot. The application rate was the same in all treatments, $100 \mathrm{~L} \mathrm{ha-1}$ and a dose of $2 \mathrm{~L} \mathrm{ha-1}$ of the product used. The first application of the product was carried out inside the nursery, and the subsequent ones, in the field after planting the seedlings, with an interval of seven days between them. Droplet spectrum analyzes were performed, in which the parameters of diameter of the volumetric median (DMV), droplet density (DEN) and cover (COB) were evaluated. The pressure at all ends influenced the coverage variable, as the pressure increases, the better the percentage of coverage. The empty cone tip MGA $60^{a}$ at a pressure of $413 \mathrm{kPa}$ promoted the best percentage of coverage of the evaluated product, therefore, the best option for application. 


\section{INTRODUÇÃO}

O café conilon (Coffea canephora Pierre), é uma cultura de grande importância no desenvolvimento do Brasil e no estado do Espírito Santo, tendo um crescimento expressivo anual em área plantada, entretanto, é uma cultura sensível ao estresse térmico e luminoso em sua fase de desenvolvimento inicial, principalmente após o transplantio das mudas em campo e uma das alternativas para minimizar os efeitos negativos desse estresse nas plantas é a utilização de compostos à base de carbonato de cálcio. Lal e Sahu (2017) relatam que o carbonato de cálcio é um mineral altamente reflexível, com formulação líquida e quando aplicado nas plantas, forma uma fina camada que reflete parte da luz, protegendo o tecido vegetal de altas intensidades da radiação solar, mas sem impedir a ocorrência de fotossíntese (Ahmed, Shaaban, \& Abd El-Aal, 2011). Da Silva et al. (2019), concluíram que a aplicação de carbonato de cálcio em mudas de café conilon obteve níveis similares ou até superiores na manutenção da transpiração e do resfriamento, quando comparados ao sombreamento natural.

Um filme de partícula de carbonato de cálcio eficaz para a aplicação em tecidos vegetais deve apresentar características importantes, como por exemplo, ser uma partícula quimicamente inerte; apresentar um diâmetro de partícula menor que $2 \mu \mathrm{m}$; e facilidade para criar um filme sobre a superfície de modo que proteja, mas não interfira nas trocas gasosas (Glenn \& Puterka, 2005). Além dessas características, a qualidade de cobertura do alvo na tecnologia de aplicação pode estar relacionada com outros aspectos, sendo os principais o diâmetro de gotas, volume de calda, pressão de pulverização, arquitetura das plantas e aos fatores climáticos. As pontas de pulverização também apresentam grande importância no processo de pulverização, sendo responsáveis pela formação das gotas e determinantes na eficiência da aplicação. A escolha correta do tipo de ponta de pulverização irá garantir uma aplicação eficiente do produto, onde gotas menores proporcionam melhor cobertura e capacidade de penetração, e gotas maiores, garantem uma aplicação com menor risco de deriva, porém, menor cobertura e penetração. Desse modo, a escolha correta do tipo de ponta de pulverização é um fator determinante na eficiência da aplicação (Ferreira, Lohmann, Campos, Viel, \& Figueiredo, 2011; Chechetto et al., 2013; Vitória \& Campanharo, 2016).

Os tipos e modelos de pontas de pulverização estão presentes no mercado em grande diversidade e com especificações técnicas para cada tipo de aplicação (tipo e modo de ação dos produtos, qual o seu alvo e condições meteorológicas). Entre as opções de pontas mais utilizadas para as pulverizações, estão as de jato plano (tipo leque) e jato cônico (cone vazio e cone cheio), na qual se diferenciam principalmente pelo ângulo e pressão de abertura do jato (Griesang et al., 2017; Júnior, 2018).

Outro fator importante para a aplicação do produto é a pressão de trabalho de uma pulverização. Esse fator possui relação direta com a sua eficácia, pois quando associada com a ponta de pulverização e condições ambientais, determinam o tamanho da gota, e consequentemente, a densidade e cobertura de gotas no alvo (Camara, Santos, Silva, Ferreira, 2008). De acordo com Nuyttens, Baetens, Schampheleire e Sonck (2007), uma combinação adequada de ponta de pulverização e pressão de trabalho pode maximizar a cobertura do alvo. Os três principais parâmetros que são mais utilizados para caracterizar o espectro de gotas das 
pulverizações são: o diâmetro mediano volumétrico (DMV) que divide o volume pulverizado em duas metades: $50 \%$ do volume têm gotas menores do que o DMV e 50\% do volume pulverizado têm gotas maiores do que o DMV (Andef, 2010); densidade de gotas (quantidade de gotas por $\mathrm{cm}^{2}$ ); e porcentagem de cobertura do alvo.

A eficiência do produto está diretamente relacionada com a tecnologia de sua aplicação, pelo fato de precisar gerar uma cobertura total sobre a superfície da folha, entretanto, ainda são escassos os dados sobre a tecnologia de aplicação desses compostos, sendo incipientes os estudos sobre a sua tecnologia de aplicação em mudas de café conilon visando uma boa eficácia do produto. Bem como são escassos os trabalhos que associem a utilização do carbonato de cálcio como protetor solar, associado com a tecnologia de aplicação. Com base nestas informações, este trabalho teve como objetivo determinar qual ponta de pulverização e pressão de trabalho irá promover uma combinação ideal para melhorar a distribuição das gotas do carbonato de cálcio em mudas de café conilon.

\section{Metodologia}

O experimento foi realizado nos meses de janeiro a maio de 2020 e conduzido em dois locais distintos, ambos em condição de campo. Inicialmente no viveiro comercial Aliança, localizado no distrito de Jacupemba, município de Aracruz-ES, e posteriormente no município de São Mateus-ES na Fazenda Experimental da Universidade Federal do Espírito Santo Campus São Mateus, com uma latitude $18^{\circ} 40^{\prime} 25^{\prime \prime} \mathrm{S}$, longitude $40^{\circ} 51^{\prime} 23^{\prime \prime} \mathrm{W}$. De acordo com a classificação climática de Köppen, o clima de ambas as regiões é caracterizado como quente e úmido, tipo Aw, com estação seca no outono-inverno e estação chuvosa na primavera-verão (Alvares et al., 2013).

Durante todo o experimento os parâmetros climáticos foram monitorados, entre eles, os dados de precipitação, temperatura e radiação, que foram obtidos pela estação meteorológica automática do Instituto Nacional de Meteorologia, localizada em São Mateus (Figuras 1 e 2).

Figura 1. Dados de precipitação $(\mathrm{mm})$ e temperatura máxima e mínima $\left({ }^{\circ} \mathrm{C}\right)$ durante o período de execução do experimento no município de São Mateus

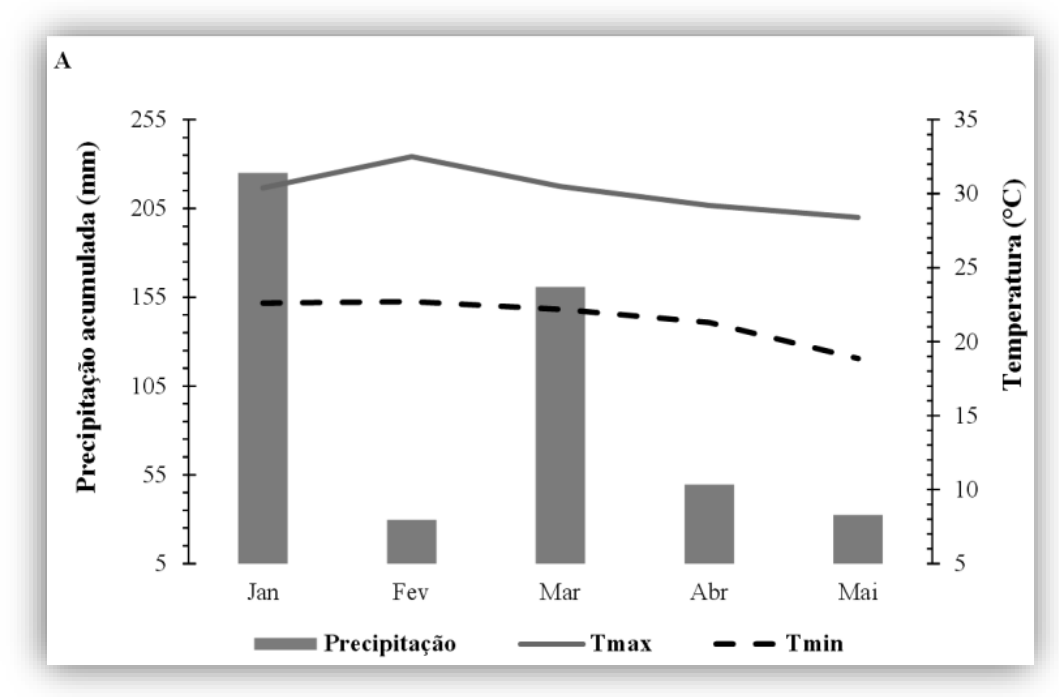

Fonte: Autores, 2021 
Figura 2. Dados de média mensal da radiação $\left(\mathrm{MJ} \mathrm{m}^{-2}\right)$ durante o período de execução do experimento no município de São Mateus

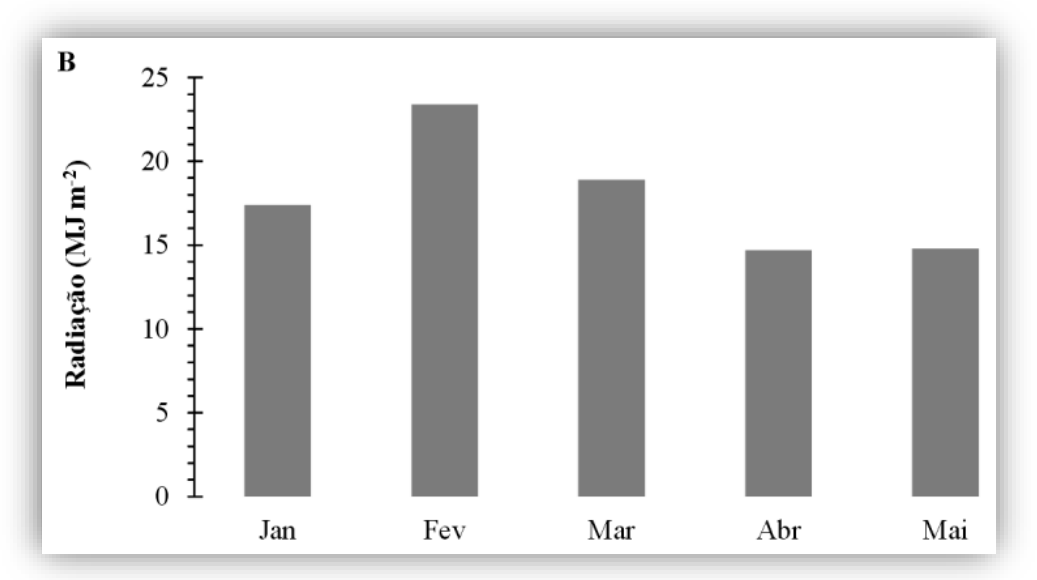

Fonte: Autores, 2021

Foi utilizado um fertilizante mineral composto por carbonato de cálcio e óxido de zinco (produto comercial Sombryt ${ }^{\circledR}$ da empresa Lithoplant), onde a sua formulação garantia um conteúdo de cálcio total $(\mathrm{Ca}): 18,5 \%$ e zinco total $(\mathrm{Zn}): 0,5 \%$, com a aplicação de uma dose de 2 litros por hectare.

$\mathrm{O}$ experimento foi conduzido em delineamento de blocos casualizados em um esquema fatorial $3 \times 4$, com quatro repetições. As fontes de variação foram as três pontas de pulverização (BD 01, MGA $60^{\circ}$ 1,0, MCP 1,5) e quatro valores de pressão de pulverização $(206,275,344$ e $413 \mathrm{kPa})$, conforme descrito nas Tabelas 1 e 2 . A taxa de aplicação foi a mesma em todos os tratamentos, $100 \mathrm{~L} \mathrm{ha}^{-1}$. O pulverizador utilizado foi o costal do tipo pressurizado por $\mathrm{CO}_{2}$, do fabricante Herbicat ${ }^{\circledR}$, com uma altura de aplicação de $0,50 \mathrm{~m}$ da superfície foliar.

Tabela 1. Especificação das pontas avaliadas

\begin{tabular}{cccc}
\hline Ponta & Tipo de jato & Fabricante & Classe de gotas \\
\hline BD 01 1,0 & Jato plano & MagnoJet & Média \\
MGA 60 $^{\circ} 1,0$ & Jato cônico vazio & MagnoJet & Fina \\
MCP 1,5 & Jato cônico vazio & MagnoJet & Fina \\
\hline
\end{tabular}

Fonte: Autores, 2021

Tabela 2. Caracterização dos tratamentos utilizados

\begin{tabular}{ccc}
\hline Tratamento & Ponta & Pressão de trabalho (kPa) \\
\hline $\mathbf{1}$ & BD 01 & 206 \\
$\mathbf{2}$ & BD 01 & 275 \\
$\mathbf{3}$ & BD 01 & 344 \\
$\mathbf{4}$ & BD 01 & 413 \\
$\mathbf{5}$ & MGA 60 01 & 206 \\
$\mathbf{6}$ & MGA 60 01 & 275 \\
$\mathbf{7}$ & MGA 60 01 & 344 \\
$\mathbf{8}$ & MGA 60 01 013 \\
$\mathbf{9}$ & MCP 1,5 & 413 \\
$\mathbf{1 0}$ & MCP 1,5 & 206 \\
$\mathbf{1 1}$ & MCP 1,5 & 275 \\
$\mathbf{1 2}$ & MCP 1,5 & 344 \\
\hline
\end{tabular}

Nota. kPa: Kilopascal. Fonte: Autores, 2021

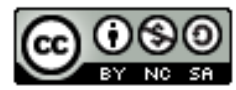


O material genético utilizado foi o clone 153 do Instituto Capixaba de Pesquisa, Assistência Técnica e Extensão Rural (Incaper), classificado como sensível ao estresse térmico e luminoso. As mudas de café foram adquiridas em um viveiro comercial certificado, com mudas padronizadas e com tratamentos nutricionais e fitossanitários regularmente utilizados no viveiro.

Uma prática utilizada na comercialização das mudas consideradas padrão pelos viveiristas é o processo de aclimatação antes de irem para o campo. No entanto, as mudas utilizadas no experimento não passaram por esse processo (apenas a testemunha). A primeira parte do experimento foi conduzida dentro do viveiro, onde sete dias antes da aclimatação que o viveirista iria proceder nas suas mudas padrão, foi realizada a primeira aplicação do carbonato de cálcio nas mudas dos tratamentos, onde permaneceram até o momento da "aclimatação", quando as mudas foram retiradas do local e levadas para a Fazenda Experimental da UFES para a realização da segunda parte do experimento.

Em campo, as mudas foram transplantadas para vasos plásticos com volume de $5 \mathrm{~L}$, preenchidos com terra, devidamente corrigidos com calcário e adubados com o adubo super simples (formulação 0015 00) conforme a recomendação de plantio. Foram realizadas outras três aplicações do formulado, com intervalos de sete dias entre elas na área experimental em campo.

Para a realização das aplicações, seguiu-se a metodologia descrita na Norma ISO 22866, International Organization for Standardization (ISO) (2005). Esta norma preconiza que durante as aplicações a temperatura deve estar entre 5 e $35^{\circ} \mathrm{C}$. Já a velocidade do vento, a norma permite que no máximo $10 \%$ das medidas estejam abaixo de $1,0 \mathrm{~m} \mathrm{~s}^{-1}$ e a direção do vento dentro de um limite de $90^{\circ} \pm 30^{\circ}$ em relação à linha de pulverização. Assim, o sentido ideal do vento para que as aplicações fossem feitas deveria ser leste-sudeste $\left(112,5^{\circ}\right)$, e podendo estar entre leste e sudeste $\left(90^{\circ}\right.$ e 135$)$.

Para a avaliação do espectro de gotas da calda, foi colocada uma etiqueta de papel hidrossenssível $(76 \times 26 \mathrm{~mm})$ em uma planta de cada tratamento. Após a aplicação, as etiquetas foram recolhidas e digitalizadas por meio de um scanner de 300 dpi de resolução na impressora multifuncional "HP Photosmart D110 Series" e submetidas às análises de cobertura, DMV e densidade de gotas pelo software computacional "Image Tool" versão 3.0.

Os dados foram submetidos ao teste de normalidade de Shapiro Wilk. Em seguida, a análise de variância pelo teste $\mathrm{F}$, sendo constatada diferença significativa, as médias das características avaliadas foram comparadas pelo teste Tukey a 5\% de probabilidade. Todas as análises foram realizadas utilizando o programa Software R CORE TEAM versão 3.0.2 (2013).

\section{RESUlTADOS E DISCUSSÃo}

O espectro de distribuição de gotas usando as pontas BD 01, MGA $60^{\circ}$ 1,0, e MCP 1,5 com diferentes pressões de pulverização são mostrados nas Tabelas 3, 4 e 5. Com relação às variáveis de cobertura e densidade de gotas, foi observado que não houve interação significativa entre os fatores, o que caracteriza a independência dos mesmos (Tabelas 3 e 4). 
Diferente da variável DMV na qual a interação entre os fatores pontas e pressões de pulverizações, foram significativas, o que indica dependência dos mesmos (Tabela 5).

Na variável cobertura para ambas as pontas, foi observado que conforme aumentou a pressão, houve um crescimento na porcentagem de cobertura (Tabela 3). Na pressão mínima de 206 $\mathrm{kPa}$ obteve-se uma cobertura de 51,62\%, já na pressão máxima de $413 \mathrm{kPa}$ obteve-se uma cobertura de 70,06\%. Esse resultado é devido ao aumento da pressão de trabalho, uma vez que quando isto ocorre, o tamanho de gotas diminui promovendo uma maior porcentagem de cobertura. Comportamento também observado por Vitória et al. (2014), em pontas de pulverização hidráulicas do tipo jato cônico vazio.

Viana et al. (2010), em seus estudos com pressões de 200, 300 e $400 \mathrm{kPa}$, observaram que o incremento na pressão proporcionou maior cobertura do alvo. Essa pressão de pulverização influencia no tamanho das gotas, uma vez que quanto maior a pressão, menor será o tamanho das gotas, promovendo assim melhores resultados de cobertura (Camara et al., 2008). Dessa forma, a ponta MGA $60^{\circ}$ comprovou sua superioridade em relação às pontas $\mathrm{BD} 01 \mathrm{e} \mathrm{MCP}$ 1,5, respectivamente para a variável cobertura. Embora na pressão de $413 \mathrm{kPa}$ a ponta MGA $60^{\circ}$ tenha mostrado uma superioridade em relação a cobertura, é importante salientar que coberturas muito altas em condições inadequadas para pulverização podem promover o escorrimento do produto para o solo. Segundo Silva (2013), as pontas tipo cone vazio são muito utilizadas na cafeicultura para aplicações foliares em função da boa densidade e cobertura proporcionadas, corroborando com os resultados obtidos.

Tabela 3. Médias da característica de cobertura (\%) de pontas de pulverização, em função de diferentes pressões

\begin{tabular}{cc}
\hline \multicolumn{2}{c}{ COBERTURA (\%) } \\
\hline \multicolumn{1}{c}{ Ponta } \\
\hline BD 01 & 47,78 b \\
MGA 60 & 80,36 a \\
MCP 1,5 & 50,23 b \\
\hline \multicolumn{2}{c}{ Pressão (kPa) } \\
\hline $\mathbf{2 0 6}$ & 51,62 b \\
\hline $\mathbf{2 7 5}$ & 56,86 b \\
\hline $\mathbf{3 4 4}$ & 59,29 b \\
\hline $\mathbf{4 1 3}$ & 70,06 a \\
\hline CV= 15,61\% & W: 0,736
\end{tabular}

Nota. Fonte: Do autor. Médias seguidas das mesmas letras minúsculas na coluna não diferem estatisticamente entre si pelo teste Tukey ao nível de 5\% de probabilidade. A ausência de letras indica que não houve significância no teste $\mathrm{F}$.

A variável densidade de gotas apresentou um comportamento inversamente proporcional à variável cobertura. As pontas que apresentaram as melhores porcentagens de cobertura evidenciaram os menores valores de densidade de gotas (Tabela 4). A pressão não teve influência para essa variável, porém, a pressão de $413 \mathrm{kPa}$ apresentou os menores valores de densidade de gotas. As pontas BD 01, MGA $60^{\circ}$ e MCP 1,5 obtiveram a densidade de 87,92; 30,71 e 67,98 gotas $/ \mathrm{cm}^{2}$, respectivamente. Esse comportamento não é comum, como citado por Matthews, Bateman e Miller (2016), pois quanto menor o diâmetro de gotas, maior será a 
densidade de gotas, consequentemente aumenta a quantidade de calda no alvo, aumentando a cobertura.

A ponta BD 01 que é classificada pelo catálogo como gotas médias, foi a que proporcionou o maior valor de densidade de gotas e menor cobertura, inversamente proporcional as pontas MCP 1,5 e MGA $60^{\circ}$, respectivamente, na qual são classificadas como gotas finas. As pontas MGA $60^{\circ}$ e MCP 1,5 formaram gotas finas, de modo que ao processar as etiquetas as mesmas se sobrepuseram, resultando na leitura uma aglomeração de gotas ao invés de cada gota individualmente, resultando assim em densidades menores, coberturas maiores e DMV maiores, respectivamente para as pontas, e o inverso para a ponta BD 01 (Tabela 5).

Cunha, Farnese e Olivet (2013), afirmam que a maior densidade de gotas está relacionada com a sensibilidade do programa em realizar a separação das gotas sobrepostas. Esta relação inversa entre densidade de gotas e DMV também pôde ser observada quando foram comparadas diferentes pontas de jato plano (Souza, Cunha, Pavanin, 2012).

Tabela 4. Médias da característica densidade de gotas $\left(\right.$ gotas $\left./ \mathrm{cm}^{2}\right)$ de pontas de pulverização em função de diferentes pressões

\begin{tabular}{|c|c|}
\hline \multicolumn{2}{|c|}{ DENSIDADE (gotas $\left./ \mathbf{c m}^{2}\right)$} \\
\hline & \\
\hline BD 01 & $87,92 \mathbf{a}$ \\
\hline MGA $60^{\circ}$ & $30,71 \mathbf{c}$ \\
\hline МCP 1,5 & $67,98 \mathbf{b}$ \\
\hline $\mathrm{CV}=35,87 \%$ & W: 0,065 \\
\hline
\end{tabular}

Nota. Fonte: Do autor. Médias seguidas das mesmas letras minúsculas na coluna não diferem estatisticamente entre si pelo teste Tukey ao nível de 5\% de probabilidade. A ausência de letras indica que não houve significância no teste $\mathrm{F}$.

O DMV é definido como o diâmetro mediano volumétrico, onde divide o volume pulverizado em duas metades iguais, no qual, em um volume de calda pulverizado de 100L/há, 50 litros obtiveram gotas com diâmetro menor que o valor de DMV, e 50 litros obtiveram gotas com diâmetro menor que o valor de DMV de cada ponta e pressão. As pontas MGA $60^{\circ}$ e MCP 1,5 são especificadas pelo fabricante como gotas finas e a ponta BD 01, é classificada como gotas médias segundo a classificação da British Crop Protection Council (Doble, Matthews, Rutherford, \& Southcombe, 1985). Essa classificação tem como parâmetro o valor de DMV, onde pontas que se enquadram em gotas finas, produzem um DMV máximo de $177 \mu \mathrm{m}$, gotas médias máximo de $218 \mu \mathrm{m}$ e gotas ultra grossas acima de $622 \mu \mathrm{m}$.

O DMV proporcionado pelas três pontas avaliadas nas pressões de 206,275 e $344 \mathrm{kPa}$ obtiveram diferença estatística entre si. Porém, em ambas as pressões e pontas, os DMVs proporcionados foram todos acima de $622 \mu \mathrm{m}$, o que as classificam como gotas ultra grossas, segundo a classificação BCPC (Doble et al., 1985). As pontas BD 01, MCP 1,5 e MGA 60 apresentaram os menores valores da variável, respectivamente. A pressão de $413 \mathrm{kPa}$ não apresentou diferença significativa entre as pontas (Tabela 5). Esses resultados podem estar associados ao método de leitura das etiquetas de papel hidrossenssível, realizado pelo software utilizado para a leitura das gotas. Foi possível observar que as pontas utilizadas promoveram gotas finas, porém, no momento da leitura das etiquetas, o programa reconheceu 
a aglomeração de gotas pequenas como uma única gota, consequentemente, resultando em valores altos de DMV, e menor densidade de gotas por $\mathrm{cm}^{2}$.

Zhu, Salyani e Fox (2011), mencionaram que os resultados de tamanho de gota e deposição obtidos através de software, podem ser imprecisos quando a porcentagem de cobertura é grande (acima de 20\%) devido à sobreposição de gotas. Hoffman e Hewitt (2005) citam que ocorrem problemas para as mensurações de gotas quando são menores do que $50 \mu \mathrm{m}$, pois estes volumes são insuficientes para fazer alteração no $\mathrm{pH}$ do papel. Com isso, podemos supor que a leitura por software podem gerar erros de leituras quando obtemos pulverizações com gotas muito finas.

Tabela 5. Médias da característica diâmetro da mediana volumétrica (DMV) de pontas de pulverização em função de diferentes pressões

\begin{tabular}{cccc}
\hline & \multicolumn{3}{c}{ DMV } \\
\hline Pressão (kpa) & BD 01 & MGA 60 $^{\mathbf{0}}$ & MCP 1,5 \\
206 & $758,25 \mathbf{b}$ & $1520,81 \mathbf{a A B}$ & $850,27 \mathbf{a b}$ \\
275 & $887,14 \mathbf{b}$ & $1619,50 \mathbf{a A}$ & $1044,22 \mathbf{a b}$ \\
344 & $876,05 \mathbf{b}$ & $1812,09 \mathbf{a A}$ & $1039,74 \mathbf{b}$ \\
413 & 1387,91 & $800,36 \mathbf{B}$ & 1353,24 \\
$\mathbf{C V = 3 4 , 9 5 \%}$ & & & $\mathbf{W} \mathbf{0 , 3 6 5}$ \\
\hline
\end{tabular}

Nota. Fonte: Do autor. Médias seguidas das mesmas letras minúsculas na linha e maiúsculas na coluna não diferem estatisticamente entre si pelo teste Tukey ao nível de $5 \%$ de probabilidade. A ausência de letras indica que não houve significância no teste F.

\section{CONCluSÃo}

A ponta cone vazio MGA $60^{\mathrm{a}}$ na pressão de $413 \mathrm{kPa}$ promoveu a melhor porcentagem de cobertura do produto avaliado, portanto, melhor opção para o produto avaliado.

A variável cobertura foi influenciada pela pressão em todas as pontas, conforme aumenta a pressão, melhor a porcentagem de cobertura.

Os resultados das variáveis de densidade de gotas e DMV podem ter sido influenciados pela capacidade de leitura do software nas etiquetas.

\section{REFERÊNCIAS}

Alvares, C. A., Stape, J. L., Sentelhas, P. C., Gonçalves, J. L. M., \& Sparovek, G. (2013). Köppen's climate classification map for Brazil. Meteorologische Zeitschrift, 22(6), 711-728. http://dx.doi.org/10.1127/0941-2948/2013/0507

Associação Nacional de Defesa Vegetal (ANDEF). (2010). Manual de Tecnologia de Aplicação de Produtos Fitossanitários. Campinas. São Paulo: Linea Creativa, 2004. Recuperado de http://www.lpv.esalq.usp.br/sites/default/files/Leitura\%20\%20Manual\%20Tecnologia\%20de\%20Aplicacao.pdf

Camara, F. T., Santos, J. L., Silva, E. A., \& Ferreira, M. C. (2008). Distribuição volumétrica e espectro de gotas de bicos hidráulicos de jato plano de faixa expandida XR 11003. Engenharia Agrícola, Jaboticabal, 28(4), 740-749. https://doi.org/10.1590/S0100$\underline{69162008000400014}$ 
Chechetto, R. G., Antuniassi, U. R., Mota, A. A. B., Carvalho, F. K., Silva, A. C. A., \& Vilela, C. M. (2013). Influência de pontas de pulverização e adjuvantes no potencial de redução de deriva em túnel de vento. Semina: Ciências Agrarias, Londrina, 34(1), 37-46. http://dx.doi.org/10.5433/1679-0359.2013v34n1p37

Companhia Nacional de Abastecimento (CONAB). (2020). Acompanhamento da Safra Brasileira. Café, Segundo Levantamento, Safra 2019. Brasília, Brasil. Recuperado de https://www.conab.gov.br/info-agro/safras/cafe/boletim-da-safra-de-cafe

Da Silva, P. O., Junior, L.F. G. O., Gonzaga, M. I. S., Sena, E. O. A., Maciel, L. B. S., Fia, M. P., Mattos, E. C., \& Carnelossi, M. A. G. (2019). Effects of calcium particle films and natural shading on ecophysiological parameters of conilon coffee. Scientia Horticulturae. 245, 171177. https://doi.org/10.1016/j.scienta.2018.10.010

Ferreira, M. C., Lohmann, T. R., Campos, A. P., Viel, S. R., \& Figueiredo, A. (2011). Distribuição volumétrica e diâmetro de gotas de pontas de pulverização de energia hidráulica para controle de corda-de-viola. Revista Planta Daninha, Viçosa, 29(3), 697-705. https://doi.org/10.1590/S0100-83582011000300024

Glenn, D. M. \& Puterka, G. J. (2005). Particle films: a new technology for agriculture. Horticultural Reviews, v.31, p.1-44. Recuperado de https://books.google.com.br/books?hl=en\&lr=\&id=iiUI7fSXXxsC\&oi=fnd\&pg=PA1\&dq=Pa rticle+films:+a+new+technology+for+agriculture. $+\& o t s=$ peUTW-r5H-

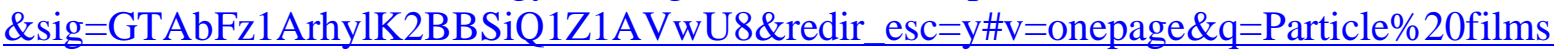
$\% 3 \mathrm{~A} \% 20 \mathrm{a} \% 20$ new \%20technology\%20for\%20agric

Griesang, F., Decaro, R. A., Santos, C. A. M., Santos, E. S., Roque, N. H. D. L., \& Ferreira, M. C. (2017). How much do adjuvant and nozzles models reduce the spraying drift? Drift in agricultural spraying. American Journal of Plant Sciences, Irvine, 8(11), 2785-2794. https://doi.org/10.4236/ajps.2017.811188

Hoffman, W. C. \& Hewitt, A. J. (2005). Comparison of three imaging systems for water sensitive papers. Applied Engineering in Agriculture, 21(6), 961-964. https://doi.org/10.13031/2013.20026

Lal, N. \& Sahu, N. (2017). Management Strategies of Sun Burn in Fruit Crops-A Review. International Journal of Current Microbiology and Applied Sciences, v.6, n.6, p.1126-1138. Recuperado de https://krishi.icar.gov.in/jspui/handle/123456789/26453. https://doi.org/10.20546/ijcmas.2017.606.131

Junior, A. M. (2018). Influência da pressão e da ponta de pulverização na distribuição de caldas em pulverizadores costais manuais (Mestrado Produção Vegetal). Universidade Estadual Paulista, Jaboticabal, SP, Brasil. Recuperado de https://repositorio.unesp.br/bitstream/handle/11449/153874/marangonijunior_a_me_jabo.pdf? sequence $=3 \&$ is Allowed $=\mathrm{y}$

Matthews, G. A., Bateman, R., \& Miller, P. (2016). Métodos de aplicação de defensivos agrícolas. 4. ed. São Paulo: Andrei.

Nuyttens, D., Baetens, K., Schampheleire, M., \& Sonck, B. (2007). Effect of nozzle type, size and pressure on spray droplet characteristics. Biosystems Engineering. 97, p.333-345. Recuperado de https://www.sciencedirect.com/science/article/pii/S1537511007000712?casa_token=Eo_D_e Oz_DcAAAAA:p3-YVj6Ym- 
N8mXiwaKKq4MN9gD1yZRyleFLH3VTFIaokkzlCKWHgYdEoUBRaGMBNvKK3CCms. https://doi.org/10.1016/j.biosystemseng.2007.03.001

R Development Core Team. (2013). $R$ : A language and environment for statistical computing. $R$ Foundation for Statistical Computing. [Software]. Vienna, Austria. Recuperado de http://www.R-project.org/

Silva, J. E. R. (2013). Deposição de calda aplicada em folhas de cafeeiro com diferentes volumes e pontas de pulverização (Dissertação de Mestrado). Universidade Federal de Viçosa, Viçosa, MG, Brasil. http://repositorio.ufu.br/bitstream/123456789/12189/1/DeposicaoCaldaAplicada.pdf

Souza, L. A., Cunha, J. P. A. R., \& Pavanin, L. A. (2012). Deposição do herbicida 2,4-D Amina com diferentes volumes e pontas de pulverização em plantas infestantes. Revista Ciência Agronômica, Fortaleza, 43(1), 78-85. Recuperado de https://www.scielo.br/j/rca/a/xp6Gt46FzjsGrZkPKBkMyQP/?format=pdf\&lang=pt.

Viana, R. G., Ferreira, L. R., Ferreira, M. C., Teixeira, M. M., Rosell, J. R. \& Machado, A. F. L. (2010). Distribuição volumétrica e espectro de gotas de pontas de pulverização de baixa deriva. Revista Planta Daninha, 28(2), 439-446. https://doi.org/10.1590/S0100$\underline{83582010000200024}$

Vitória, E. L., Neto, F. C. R., Chagas, K., Teixeira, M. M., Quirino, A. L. S., \& Santiago, H. (2014). Distribuição volumétrica e espectro de gotas de pontas de pulverização de jato cônico vazio DDC2 novas e usadas. Revista Agro@Mbiente On-Line, 8(3), 368-376. Recuperado de https://revista.ufrr.br/agroambiente/article/view/1831/1431. https://doi.org/10.5327/Z 1982$\underline{8470201400031831}$

Vitória, E. L. \& Campanharo, A. (2016). Amostra de etiquetas hidrossensíveis para determinação de espectro de gotas em pulverização no cafeeiro canephora. Coffee Science, $11(3)$ 367-373. 